as peptides in the groove of MHC class I molecules. As a consequence, priming or boosting $\mathrm{CD}^{+}$effector T cells with tumor specificity can be accomplished by blocking the CD47 checkpoint. Whether CD47 blockade-induced APC activation can also enhance $\mathrm{CD} 4^{+} \mathrm{T}$ cell immunity against cancer remains an unanswered and potentially critical question ${ }^{9}$.

What are the key milestones that still must be reached in the clinical development of the CD47 blockade as a cancer immunotherapy? First and foremost is a demonstration of safety. Although expressed broadly on a variety of tumors, CD47 is also expressed on certain healthy tissues; there is marked expression, for example, in the human cerebral cortex and cerebellum. The proposed therapeutic index of the CD47 blockade relies on the hypothesis that healthy cells lack an effective 'eat me' signal, rendering the CD47 blockade biologically silent ${ }^{2}$. However, this obviously merits careful study in phase 1 trials, especially if patients are concomitantly treated with chemotherapy or radiation, which can upregulate 'eat me' signals. Depletion of CD $47^{+}$red blood cells is an expected on-target clinical adverse effect $^{2}$, albeit one that is relatively easy to manage clinically. In another sense, however, treatment-related anemia may be a useful in vivo biomarker for the evaluation and comparison of novel CD47-directed agents.

Second, assuming that CD47 antibodies can penetrate the tumor microenvironment, achieving clinical efficacy using the CD47 blockade could be difficult in the absence of either a highly immunogenic tumor or the incorporation of other immune modulatory agents to override other immunosuppressive features of some tumors. Although it remains to be seen whether experimental neo-antigens, such as ovalbumin, mimic $\mathrm{T}$ cell neo-epitopes that can arise from somatic tumor mutations, the bulk of CD47 blockade-related preclinical data ${ }^{8-10}$ implies utility in prescreening patients for high mutational and neo-epitope burden. A similar approach to enrich for clinical responders has been proposed for checkpoint therapy. Another strategy, as outlined by Liu et al. ${ }^{6}$, is to administer chemotherapy before, but not after, treatment with CD47blocking antibodies to promote tumor death and antigen release before the activation of APCs ${ }^{6,10}$.

Given these proposed mechanisms of action, it may be beneficial to combine the CD47 blockade with agents that activate APCssuch as agonist CD 40 antibodies ${ }^{11}$ —or those that provide strong 'eat me' signals-such as anti-tumor monoclonal antibodies ${ }^{2}$. $\mathrm{T}$ cell responses, once induced in patients by a CD47 blockade, could be further enhanced with checkpoint antibodies, such as US Food and Drug Administration-approved reagents that block PD-1 or CTLA-4 checkpoints. Despite impressive clinical results in some patients, checkpoint therapy is unsuccessful in most people, mainly because of the lack of tumorspecific $\mathrm{T}$ cell priming ${ }^{12}$. Combining the therapy with anti-CD47 represents a rational candidate approach to extending the clinical range of the PD- 1 and CTLA- 4 blockades.
Finally, among the myriad of emerging immunotherapy agents for cancer, an antibody that achieves $\mathrm{T}$ cell activation by blocking a negative regulatory pathway has obvious dose and schedule pharmacological advantages over pure agonists. We have, however, entered the era of combinations. Optimal immune activation involves non-redundant pathways that work synergistically. The caution, therefore, is not to throw away CD47 blockade or novel immune agonists if the first clinical trials of monotherapy fall short of expectations.

\section{ACKNOWLEDGMENTS}

This work was supported by US National Institutes of Health grant R01 CA169123.

\section{COMPETING FINANCIAL INTERESTS}

The author declares no competing financial interests.

1. Grimsley, C. \& Ravichandran, K.S. Trends Cell Biol. 13 648-656 (2003).

2. McCracken, M.N., Cha, A.C. \& Weissman, I.L. Clin. Cancer Res. 21, 3597-3601 (2015).

3. Willingham, S.B. et al. Proc. Natl. Acad. Sci. USA 109, 6662-6667 (2012)

4. Beatty, G.L. et al. Science 331, 1612-1616 (2011).

5. Ruffell, B. \& Coussens, L.M. Cancer Cel/ 27, 462-472 (2015).

6. Liu, X. et al. Nat. Med. 21, 1209-1215 (2015).

7. Sharma, P. \& Allison, J.P. Cell 161, 205-214 (2015).

8. Barrett, D.M., Singh, N., Porter, D.L., Grupp, S.A. \& June, C.H. Annu. Rev. Med. 65, 333-347 (2014).

9. Tseng, D. et al. Proc. Natl. Acad. Sci. USA 110 11103-11108 (2013)

10. Soto-Pantoja, D.R. et al. Cancer Res. 74, 6771-6783 (2014).

11. Vonderheide, R.H. \& Glennie, M.J. Clin. Cancer Res. 19, 1035-1043 (2013).

12. Lesokhin, A.M., Callahan, M.K., Postow, M.A. \& Wolchok, J.D. Sci. Transl. Med. 7, 280sr281 (2015).

\title{
Antigen-presenting cells look within during influenza infection
}

\author{
Justine D Mintern \& Jose A Villadangos
}

\section{During viral infections, antigen-presenting cells (APC) have traditionally been thought to recruit and activate CD4 ${ }^{+}$ $T$ cells by presenting fragments of viral proteins captured from the extracellular environment. A new study indicates that the material the APCs need to present is much closer: in fact, APCs need to make it themselves.}

Humans-even immunologists-like to make sense of the world's complexity by

Justine D. Mintern is in the Department of Biochemistry and Molecular Biology, Bio21 Molecular Science and Biotechnology Institute, The University of Melbourne, Parkville, Victoria, Australia. Jose A. Villadangos is in the Department of Biochemistry and Molecular Biology, Bio21 Molecular Science and Biotechnology Institute, and in the Department of Microbiology and Immunology, Doherty Institute for Infection and Immunity, The University of Melbourne, Victoria, Australia. e-mail: j.villadangos@unimelb.edu.au establishing simple, and if possible, binary categories. For example, the discovery that the processing of extracellular antigens for presentation by major histocompatibility class II (MHC II) molecules takes place in endosomal compartments ${ }^{1}$, whereas antigens presented by MHC I molecules are produced by the cells themselves and processed in the cytosol ${ }^{2,3}$, led to the establishment of a simple, now 30-year-old paradigm: the antigens presented by MHC I are endogenous (synthesized by the cells performing the presentation) and those presented by MHC II are exogenous (synthesized by other cells).
This is a rule stated in most immunology textbooks and frequently reiterated in the introductions of primary research papers on antigen presentation. But it is a gross simplification. Cross-presentation-the process whereby exogenous antigens are presented via MHC I-has long been known to represent an important departure from the paradigm $^{4}$, but it took three decades for its physiological role to be widely accepted ${ }^{5}$. In this issue of Nature Medicine, Miller et al. present data indicating that, at least in the mouse influenza model studied, induction of robust $\mathrm{CD} 4^{+} \mathrm{T}$ cell responses requires $\mathrm{MHC}$ 


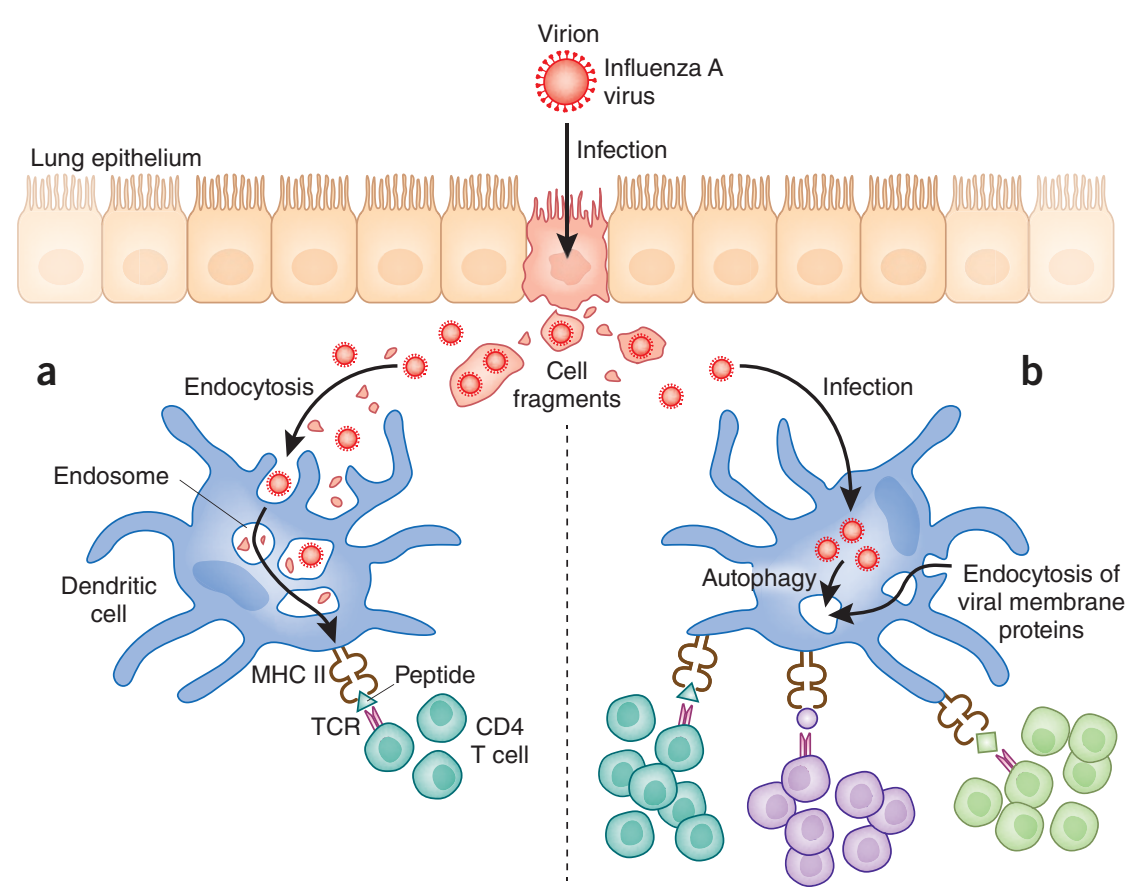

Figure 1 Influenza A virus (IAV) infects lung epithelial cells, which release virions and/or die, generating cell fragments that harbor virus antigens. (a) Dendritic cells (DCs) can capture these exogenous antigens by endocytosis (left). The DCs process the antigens in endosomal compartments and present them via $\mathrm{MHC} / \mathrm{II}$ to $\mathrm{CD} 4^{+} \mathrm{T}$ cells. The repertoire of viral peptides presented, and the magnitude of presentation, are relatively small, so the antiviral CD4+ T cell response elicited is limited. (b) IAV can also infect DCs, which produce viral proteins. These endogenous antigens access endosomal compartments by endocytosis (membrane proteins) or autophagy (cytosolic proteins) and are also presented via $\mathrm{MHC}$ II to $\mathrm{CD} 4^{+} \mathrm{T}$ cells. The viral peptides presented by this pathway are more diverse and their presentation is more efficient, leading to more vigorous antiviral CD4 ${ }^{+} \mathrm{T}$ cell responses. MHC II presentation of endogenous viral antigens by the infected DCs may involve accessory molecules that are not used by DCs performing presentation of exogenous antigens.

II presentation of endogenous antigens produced by infected $\mathrm{APCs}^{6}$. This paradigm shift has important implications for our understanding of antiviral immunity and vaccine development.

The confusion about the origins of antigens presented via MHC I as compared to II is partly semantic. The most accurate criterion to categorize the type of antigens presented by each molecule is the site of peptide production: the cytosol for MHC I and endosomal compartments for MHC II. The problem is that the term 'cytosolic' is often used interchangeably with 'endogenous' and the term 'endosomal' as synonymous with 'exogenous'. It is true that, except in the few cell types capable of crosspresentation, the vast majority of cytosolic proteins are endogenous. However, the content of endosomal compartments is always both endogenous (membrane proteins, endosomal components, cytosolic proteins delivered to endosomes by autophagy) and exogenous (endocytosed from the extracellular environment). Because the proteases that produce MHC II ligands cannot distinguish exogenous from endogenous proteins, MHC II molecules end up presenting peptides derived from both ${ }^{7}$. However, this conflation of endosomal with exogenous helped cement a common misconception in the field: that the APCs that activate $\mathrm{CD} 4^{+}$ $\mathrm{T}$ cells by presenting antigens via $\mathrm{MHC}$ II-namely, dendritic cells (DCs) - must have obtained this antigen from an exogenous source. In the case of viral infection, this exogenous source would correspond to virions or to cells infected with the virus (Fig. 1). In this scheme, the location of the viral antigen within the infected cell is irrelevant; what matters is that the antigen has been produced by a different cell from the one performing the presentation.

Miller et al. put this assumption to the test using mouse models in which the influenza A virus could infect either only non-DCs or both DCs and non-DCs. Their conclusion is that only when DCs are infected, enabling presentation of viral antigens produced by the DCs themselves, does an efficient $\mathrm{CD} 4^{+}$ T cell response develop ${ }^{6}$. Next they explored the role of known components of the MHC
II presentation machinery ${ }^{8}$ in the presentation of endogenous viral antigens in DCs. Regardless of the mechanism used to access endosomes, it is assumed that the conversion of proteins into peptides capable of binding to MHC II will be carried out by proteases and other enzymes located in the endocytic route. For example, the endosomal reductase GILT would be expected to assist proteolysis by breaking disulfide bonds contained in the antigen. Binding of the peptide antigens to MHC II molecules should be dependent on the endosomal chaperone H-2M. However, Miller et al. found that the presentation of most viral peptides derived from endogenous sources did not require H-2M or GILT. Even more surprisingly, presentation of some peptides required the proteasome and the transporter TAP, molecules involved in MHC I, not MHC II, presentation. A role for MHC I presentation machinery in MHC II presentation is not without precedent ${ }^{9}$, but its contribution in the setting of infection is not clear; additional work is needed to clarify the precise role of MHC I machinery in this process.

The conclusions of this paper raise important considerations for future studies. For example, when assessing the role of different APCs in priming $\mathrm{CD} 4^{+} \mathrm{T}$ cells against viruses, it will be important to take into account the susceptibility of each APC subtype to infection. Specializations amongst APC may also be based on differential capacity to present endogenous viral antigens via MHC II ${ }^{10}$. These new results also point to potential strategies that might be exploited by viruses to impair the induction of neutralizing antibody responses, which require $B$ cells to present antigens to the same helper $\mathrm{CD} 4^{+} \mathrm{T}$ cells that were primed by $\mathrm{DCs}{ }^{11}$. If the viral antigens are presented only by the endogenous route, and the offending virus infects DCs but not B cells, the helper CD $4^{+}$ T cells and B cells would not interact productively, and antibody production would be blunted.

Finally, there is a practical and rather unsettling implication of the conclusions of Miller et al. ${ }^{6}$ : 'dead' or inactivated viruses used in vaccine preparations may not be capable of inciting robust and protective virus-specific $\mathrm{CD}^{+} \mathrm{T}$ cell responses because, unlike live viruses, inactivated viruses will not productively infect DCs and produce endogenous antigens. To what extent are these implications correct? To how many and which viruses do they apply? These are questions that viral immunologists will now have to address by seeking the truth not only outside but also inside the APCs. 
COMPETING FINANCIAL INTERESTS

The authors declare no competing financial interests.

1. Ziegler, H.K. \& Unanue, E.R. Proc. Natl. Acad. Sci. USA 79, 175-178 (1982).

2. Townsend, A.R., Gotch, F.M. \& Davey, J. Cell 42 457-467 (1985).
3. Moore, M.W., Carbone, F.R. \& Bevan, M.J. Cell 54 777-785 (1988).

4. Bevan, M.J. J. Exp. Med. 143, 1283-1288 (1976).

5. Carbone, F.R. \& Heath, W.R. J. Immunol. 185 , 1353-1354 (2010).

6. Miller, M.A., Ganesan, A.P.V., Luckashenak, N., Mendonca, M. \& Eisenlohr, L.C. Nat. Med. 21 1216-1222 (2015).
7. Rudensky, A.Y., Preston-Hurlburt, P., Hong, S.C., Barlow, A. \& Janeway, C.A. Jr. Nature 353, 622-627 (1991).

8. Villadangos, J.A. Mol. Immunol. 38, 329-346 (2001).

9. Eisenlohr, L.C. Mol. Immunol. 55, 169-171 (2013).

10. Young, L.J. et al. Nat. Immunol. 9, 1244-1252 (2008).

11. Ramiscal, R.R. \& Vinuesa, C.G. Immunol. Rev. 252 146-155 (2013).

\title{
Remodeling the susceptibility to stress-induced depression
}

\author{
Farahnaz Sananbenesi \& Andre Fischer
}

\begin{abstract}
Depression is mechanistically not well understood. A new study investigates the expression of chromatin-remodeling complexes in a mouse model for depression and describes an epigenetic pathway that may explain why some individuals are more susceptible to stress-induced depression than others.
\end{abstract}

Depression is the leading cause of disability worldwide ${ }^{1}$, causing an enormous emotional and economic burden for individuals and society. Recently, epigenetic processes have been implicated in neuropsychiatric and neurodegenerative diseases ${ }^{2}$. These epigenetic processes, such as histone modification and DNA methylation, allow transient stimuli to induce long-term adaptive changes in gene expression through regulation of the chromatin state. An epigenetic mechanism so far relatively unexplored in neuroscience is chromatin-remodeling, an ATP-dependent process that regulates the positioning of nucleosomes across the genome and is catalyzed by chromatin-remodeling complexes ${ }^{3}$. In this issue Sun et al. ${ }^{4}$ screen the expression of chromatin-remodeling complex subunits in a mouse model of depression in response to chronic social defeat stress (CSDS). They find that altered expression of the chromatinremodeling protein BAZ1A (also known as ACF1) correlates with susceptibility to stressinduced depression.

In the chronic social defeat stress model for stress-induced depression, a mouse is attacked by another, more aggressive individual for a short time and subsequently spends the day in sensory but not physical contact with the 'bully'. Sun et al. found that after $10 \mathrm{~d}, 65 \%$ of the mice that underwent such a treatment

Farahnaz Sananbenesi and Andre Fischer are in the Research Group for Epigenetics in Neurodegenerative Diseases, German Center for Neurodegenerative Diseases (DZNE) Göttingen, Göttingen, Germany, and Andre Fischer is also in the Department of Psychiatry and Psychotherapy, University Medical Center Göttingen, Göttingen, Germany.

e-mail: afische2@gwdg.de develop depressive-like behavior, and they hence classified these as 'susceptible' to stressinduced depression. They furthermore showed that BAZ1A, a subunit of the ISWI chromatinremodeling complex, was persistently increased in the nucleus accumbens (NAc) of mice susceptible to stress-induced depression, but not in resilient mice or in mice not exposed to stress $^{4}$. The nucleus accumbens (NAc) is part of the limbic system and is thought to have a role in depression ${ }^{5}$. In line with this observation, the authors found that BAZ1A levels were elevated in postmortem human brain tissue of individuals who suffered from depression as compared to control individuals not affected with depression.

Then, using a combination of cell biological and in vivo optogenetic experiments in which light is used to specifically activate selected neurons, the authors showed that increased expression of Bazla (Acf1) in the NAc in response to CSDS is most likely due to increased release of brain-derived neurotrophic factor (BDNF) and increased firing of dopaminergic neurons projecting from the ventral tegmental area to the NAc. Indeed, overexpression of Bazla in the NAc of mice increased susceptibility for stress-induced depressive-like behavior, whereas genetic knockdown of Bazla increased resilience, providing convincing evidence that BAZ1A is a key regulator of depressive-like behavior.

Because BAZ1A is part of a chromatin-remodeling complex, the authors then sought to identify which genes are regulated by BAZ1Adependent mechanisms. To this end, they carried out chromatin-immunoprecipitation followed by sequencing (ChIP-Seq) and found that in the Nac, the number of BAZ1A genomic binding sites in mice susceptible to stressinduced depression was more than double that in resilient mice. The authors compared these data to the positioning of histone $\mathrm{H} 3$ across the genome of cells from the NAc and observed that increased BAZ1A binding in susceptible as compared to resilient animals was associated with a lack of well-defined nucleosome-depleted transcription start sitewhich is a signature of active genes. This data suggested that, in susceptible mice, increased BAZ1A binding would cause repression of the corresponding target genes. Indeed, Sun et al. found, for a number of selected genes, that some but not all of those that showed increased BAZ1A binding upon CSDS also show decreased expression ${ }^{4}$. Among these genes, they selected two that have been linked to depression and synaptic plasticity ${ }^{6-8}-A g \operatorname{tr} 1 b$, encoding the angiotensin II receptor, and Rab3a, encoding Rasassociated protein $3 \mathrm{a}$-for further analysis and demonstrate that their overexpression can partially reverse depressive-like behavior in susceptible animals.

The data presented in this study is interesting on several levels. The authors demonstrate that BAZ1A-mediated chromatin remodeling is a novel regulator of stress-induced depressive behavior (Fig. 1). In future studies it will be interesting to see what mechanisms induce the persisting increase of BAZ1A levels brought about by stress.

In addition, as for all multifactorial neuropsychiatric diseases the identification of molecular pathways that allow patient stratification and thus may eventually help to develop personalized treatments is a key issue for future research. Thus, that in mice the BAZ1A chromatin-remodeling pathway apparently distinguishes individuals that are susceptible to stress-induced depression from those that are not could be used to personalize 